\title{
Prolonged Habituation of the Gill-Withdrawal Reflex in Aplysia Depends on Protein Synthesis, Protein Phosphatase Activity, and Postsynaptic Glutamate Receptors
}

\author{
Youssef Ezzeddine ${ }^{1}$ and David L. Glanzman ${ }^{1,2}$ \\ ${ }^{1}$ Department of Physiological Science and ${ }^{2}$ Department of Neurobiology and the Brain Research Institute, David Geffen School of Medicine, University of \\ California, Los Angeles, Los Angeles, California 90095
}

Despite representing perhaps the simplest form of memory, habituation is not yet well understood mechanistically. We used a reduced preparation to analyze the neurobiological mechanisms of persistent habituation of a simple behavior, the defensive withdrawal reflex of the marine snail Aplysia californica. This preparation permits direct infusion of drugs into the abdominal ganglion during training via a cannula in the abdominal artery. Using siphon-elicited gill withdrawal, we demonstrate habituation of withdrawal that persists for $1-6 \mathrm{hr}$ after repeated, spaced blocks of habituating stimulation. This form of habituation exhibits site specificity and requires protein synthesis because it is blocked by the presence of anisomycin, a protein synthesis inhibitor. We also find that habituation of gill withdrawal requires protein phosphatase activity, because it is blocked by okadaic acid, an inhibitor of protein phosphatase. Finally, habituation of gill withdrawal requires activation of NMDA-type and AMPA-type postsynaptic receptors within the abdominal ganglion, because it is blocked by infusion of DL-2-amino-5-phosphonovaleric acid or 6,7-dinitroquinoxaline-2,3-dione. The requirement for activation of postsynaptic glutamatergic receptors indicates that homosynaptic depression, an exclusively presynaptic mechanism that has been implicated previously in habituation in Aplysia, does not play a significant role in persistent habituation of the withdrawal reflex. Our results indicate that postsynaptic mechanisms, possibly including modulation of glutamate receptor function, play a major, heretofore unsuspected, role in habituation in Aplysia.

Key words: Aplysia; habituation; long-term memory; protein synthesis; protein phosphatase; okadaic acid; APV; NMDA; AMPA; DNQX; glutamate receptors; postsynaptic processes, LTD

\section{Introduction}

Habituation, perhaps the simplest form of learning, is the decrement in an animal's response to repeated presentations of a (typically innocuous) stimulus, in which the decrement cannot be ascribed simply to injury, receptor adaptation, or motor fatigue (Thompson and Spencer, 1966). Habituation is a nearly universal form of learning, having been observed in animals ranging from roundworms (Rankin et al., 1990) to humans (Geer, 1966). Despite its apparent simplicity and universality, our understanding of the neurobiological basis of habituation remains incomplete. A generation ago, it was widely agreed that habituation, at least for certain simple reflexes in invertebrate organisms, was attributable to a presynaptic mechanism: homosynaptic depression of transmitter release from the terminals of the sensory neurons that mediate the reflexes (Zucker, 1972; Castellucci and Kandel, 1974; Krasne, 1976; Kandel, 1978). However, more recent work has

Received May 12, 2003; revised Aug. 15, 2003; accepted Aug. 28, 2003.

This work was supported by National Science Foundation Grant IBN-9808930 and National Institutes of Health Grant NS29563 (D.L.G.). We thank Drs. Frank Krasne, Marc Klein, Tom 0'Dell, and Adam Roberts for their helpful comments on this manuscript.

Correspondence should be addressed to Dr. David L. Glanzman, Gonda (Goldschmied) Neuroscience and Genetics Research Center, 695 Young Drive South, Box 951761, University of California, Los Angeles, Los Angeles, CA 90095 1761. E-mail: dglanzman@physci.ucla.edu.

Copyright $\odot 2003$ Society for Neuroscience $\quad$ 0270-6474/03/239585-10\$15.00/0 indicated that inputs from extrinsic modulatory pathways are critical to the full expression of behavioral habituation, even in invertebrates (Krasne and Glanzman, 1995; Krasne and Teshiba, 1995; Stopfer and Carew, 1996).

Habituation is commonly observed to have both short-term and long-term forms. Cellular correlates of long-term habituation (LTH) of the defensive withdrawal reflex of Aplysia (Carew et al., 1972; Carew and Kandel, 1973) have been identified. Behavioral training that results in habituation persisting for $\geq 24 \mathrm{hr}$ causes a long-term depression of transmission as sensorimotor synapses (Castellucci et al., 1978), as well as a significant reduction in active zones and varicosities associated with sensory neurons in the abdominal ganglia of habituated animals (Bailey and Chen, 1983, 1988).

Although the cellular expression of LTH of the withdrawal reflex in Aplysia is attributable, at least in part, to long-term presynaptic changes, the locus of the induction of the cellular changes is uncertain. Are the long-term changes in sensory neuron transmitter release and structure that are associated with LTH induced, as well as expressed, presynaptically? Or, rather, are the long-term cellular changes induced by postsynaptic processes? Here, we show that postsynaptic mechanisms, particularly activation of postsynaptic glutamate receptors, play a critical role 


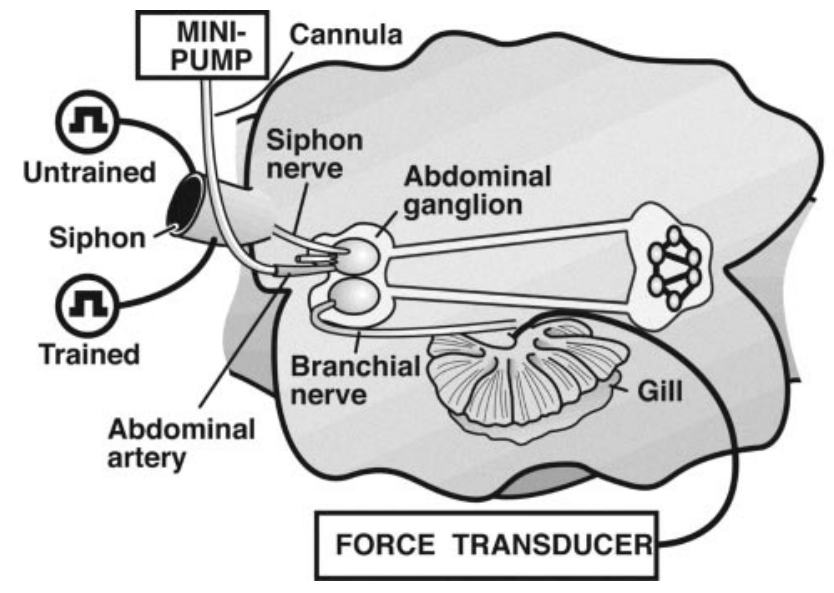

Figure 1. Reduced preparation of Aplysia used for experiments investigating habituation of siphon-elicited gill withdrawal. The abdominal ganglion is shown artificially enlarged relative to the other central ganglia. The preparation shown is that used for the within-preparation experiments. The preparation used for the between-preparation experiments (Fig. 2) was similar, but only one site on the siphon was stimulated.

in inducing long-lasting habituation of the withdrawal reflex in Aplysia.

The habituation we report here persists for $1-6 \mathrm{hr}$ after the last block of stimuli and depends on protein synthesis (see Results). A requirement for protein synthesis has been typically regarded as an essential characteristic of long-term memory (Davis and Squire, 1984; Goelet et al., 1986). However, we decided not to refer to the present form of habituation as long term. Previous studies of LTH in Aplysia have reported habituation that persisted for $24 \mathrm{hr}$ to weeks (Carew et al., 1972; Carew and Kandel, 1973; Castellucci et al., 1978; Stopfer et al., 1996), and it is possible that LTH of the duration achieved in these previous studies will ultimately prove distinct, mechanistically, from the habituation we demonstrate here. We will therefore refer to the prolonged habituation shown in the present experiments as "longlasting habituation (LLH)."

Parts of this work have been published previously in abstract form (Ezzeddine and Glanzman, 2001, 2002).

\section{Materials and Methods}

Animals. Adult Aplysia californica (75-150 gm) were obtained from a local supplier (Alacrity Marine, Redondo Beach, CA). Animals were housed in a 50 gallon aquarium filled with cooled $\left(14^{\circ} \mathrm{C}\right)$, aerated artificial seawater (ASW) (Instant Ocean; Aquarium Systems, Mentor, $\mathrm{OH}$ ). All animals were housed for at least $24 \mathrm{hr}$ before the start of the experiment.

Preparation. An animal was initially anesthetized with an injection of $60-80 \mathrm{cc}$ of isotonic $\mathrm{MgCl}_{2}$ into the animal's foot. The animal was then placed, ventral side up, in a dissection tray. A longitudinal incision was made along the length of the foot. The body wall was pinned back to expose the digestive organs and nervous system. The digestive system was removed to gain access to the nervous system. The CNS was drawn toward the tail, and most of the peripheral nerves were cut. The head of the animal, together with the buccal ganglia, was then removed. The rest of the animal, including the mantle shelf, gill, and siphon, together with the tail and the entire CNS (minus the buccal ganglia), was preserved (Fig. 1). The CNS was left connected to the siphon and gill via the siphon and branchial nerves of the abdominal ganglion. The animal was transferred to another dissection tray filled with $50 \% \mathrm{MgCl}_{2}-50 \%$ normal ASW. The artery leading to the abdominal ganglion was cannulated with polyethylene tubing ( 0.024 outer diameter, 0.011 inner diameter; Intramedic, Parsippany, NJ), which was connected to a peristaltic pump (P720; Instech, Plymouth Meeting, PA). During experiments, the abdominal ar- tery was perfused with aerated ASW $\left(15^{\circ} \mathrm{C}\right)$ via the cannula at a rate of $\sim 1$ $\mathrm{ml} / \mathrm{hr}$ until the start of the experiment (onset of the pretests), at which point the perfusion rate was decreased to $0.5 \mathrm{ml} / \mathrm{hr}$. This perfusion rate was then maintained throughout the experiment. The cannula in the abdominal artery was also used to selectively administer drugs to the abdominal ganglion (below). After cannulation of the abdominal artery, the preparation was transferred to a Lucite experimental chamber filled with normal ASW and pinned, dorsal side up, to the Sylgard-lined bottom of the chamber. The siphon was not pinned but was left to move freely. The afferent vein of the gill was cannulated with polyethylene tubing (above) and perfused with chilled, aerated ASW via a peristaltic pump (Dynamax RP1; Rainin, Oakland, CA) at a rate of $100 \mathrm{ml} / \mathrm{hr}$. The cannula in the afferent vein was secured with a surgical silk suture, and a force transducer (model 1040 or 1030, ADInstruments, Grand Junction, CO) was connected to the suture with a small metal hook. The force produced by movement of the transducer was calibrated by hanging gram weights from the metal hook. Habituating stimuli (see below) were delivered to the siphon via pairs of Teflon-insulated platinum wires (0.005 mm in diameter; catalog \#773000; A-M Systems, Carlsborg, WA). The uninsulated tips of the wires were inserted into the siphon at its base. In the within-preparation experiments (see below), a platinum wire was inserted into each side of the siphon, and a ground wire was placed in the bath. The rest of the preparation was perfused with normal ASW (13$15^{\circ}$ ) at a rate of $11 / \mathrm{hr}$ throughout the experiment.

After the intensity of the siphon stimuli to be used for an experiment had been determined (see below), the preparation was rested for $2 \mathrm{hr}$, during which time the afferent vein of the gill and the rest of the preparation were perfused with normal ASW to completely wash out the $\mathrm{MgCl}_{2}$. This perfusion was maintained for the rest of the experiment. At the end of the $2 \mathrm{hr}$ rest period, a single stimulus (at the predetermined intensity for habituation) was delivered to the siphon. Preparations that did not respond to this initial stimulus with a visible gill contraction were discarded. Furthermore, data from preparations from the experiments in which a within-preparation design was used (below) were only accepted if the posttest response of the "untrained" site was at least $80 \%$ of the pretest response (see Experimental design).

Shipments of Aplysia from our local supplier varied significantly according to whether or not preparations from the animals expressed longlasting habituation. In some cases, these differences were seasonal and may represent differences in the behavioral state of the animals. In other cases, the differences may have been related to the health of the animals. Because of the variability in the amount of habituation exhibited by preparations from different shipments of animals, we were obliged to institute a control criterion to ensure that animals in a given shipment were capable of expressing LLH. On the basis of the results of pilot experiments, we decided that preparations could be considered to have undergone long-lasting habituation if the posttest response (at either $6 \mathrm{hr}$ or $60 \mathrm{~min}$ after the end of training; see below) of the "trained" site to the habituating stimulus was $\leq 70 \%$ of the pretest response. Using this criterion, we ran an initial control experiment with one animal from each shipment. If that control preparation did not habituate by $\geq 30 \%$, the entire shipment of animals was rejected.

Siphon stimulation. Both habituating and test stimuli were brief trains ( $500 \mathrm{msec}, 25 \mathrm{~Hz}$ ) of current pulses (10 msec duration) generated by an electronic stimulator (S48/S88; Grass Instruments, Quincy, MA); the stimuli were delivered to the siphon via implanted platinum wires (see above). The intensity of the stimuli was calibrated for each preparation to be just suprathreshold for reliably eliciting gill withdrawal. The intensity of the stimuli applied to the untrained site in experiments using the within-preparation design (below) was determined in the same way and was also fixed for a given experiment.

Drugs. All drugs were purchased from Sigma-Aldrich (St. Louis, MO). The drugs were dissolved in either normal ASW or DMSO as stated in Results. The drugs were infused directly into the abdominal ganglion via a cannula in the abdominal artery (above). The anisomycin was infused into the abdominal ganglion for $45 \mathrm{~min}$ to $1 \mathrm{hr}$ before the onset of the pretests; okadaic acid (OA) and 1-nor-okadone were infused for $2 \mathrm{hr}$ before the onset of the pretests. Infusion of anisomycin, okadaic acid, and 1-nor-okadone was maintained throughout those experiments in which 
they were used. We performed two sets of DL-2-amino-5-phosphonovaleric acid (APV) experiments. In the first set of experiments, APV was infused into the abdominal ganglion for $45 \mathrm{~min}$ to $1 \mathrm{hr}$ before the onset of the pretests and was present throughout the experiments, as in the experiments involving anisomycin, okadaic acid, and 1-nor-okadone. In the second set of experiments, APV was present only during training; both the pretests and posttests were performed in ASW. Our results for these two sets of APV experiments were very similar (see Results). (Notice that both okadaic acid and 1-nor-okadone took a prolonged period of time for wash in, so it was not feasible to have these compounds present only during training. We did not perform experiments with the protein synthesis inhibitor present only during training.) 6,7Dinitroquinoxaline-2,3-dione (DNQX) was infused at a rate of $1 \mathrm{ml} / \mathrm{hr}$ starting immediately after the pretests; it was then washed out of the abdominal ganglion with normal ASW after the end of habituation training.

Experimental design. There were two basic experimental designs. In our initial set of experiments (between-preparation design), there were two separate groups of preparations: an experimental group that received habituation training and a control group that received only test stimuli. In subsequent experiments, we used a modified design in which each preparation served as its own control (within-preparation design). In the experiments using the within-preparation design, two different sites on the siphon were stimulated: one of the sites (trained) received habituation training, whereas the other site (untrained) received only test stimuli (Fig. 1). In the between-preparation experiments, each preparation initially received three pretests at a rate of one per $10 \mathrm{~min}$. Fifteen minutes after the third pretest, some preparations (trained) received habituation training. The training protocol consisted of four blocks of 20 stimuli [interstimulus interval (ISI) of $30 \mathrm{sec}$ ]. There was a $90 \mathrm{~min}$ rest period between each block of habituating stimuli. After the fourth training block, there was a $6 \mathrm{hr}$ rest period, and then the preparation received three posttests at a rate of one per $10 \mathrm{~min}$. Control (untrained) preparations received three posttests at the same rate $12 \mathrm{hr}$ after the pretests. In the within-preparation experiments, the side of the siphon that was chosen to be the trained site was alternated systematically between left and right sides. The trained site initially received three pretests at a rate of one per $10 \mathrm{~min}$. Ten minutes after the third pretest to the trained site, the other side of the siphon (untrained site) received a single pretest stimulus. Fifteen minutes after the pretest stimulus to the untrained site (25 min after the third pretest to the trained site), the trained site received habituation training. The training protocol was identical to that used for the between-preparation experiments. In the within-preparation experiments, there was $60 \mathrm{~min}$ rest period between the final block of training stimuli and the onset of the posttests. Then, the trained site received three posttests at one per $10 \mathrm{~min}$. Ten minutes later, the untrained site received a single posttest.

A somewhat different within-preparation experimental protocol was used in one set of the experiments with the drug APV and in all of those with the drug DNQX (see Results). The altered protocol was used to ensure that the drugs were only present during training and were completely washed out after training. In these experiments, the trained site received two pretests at one per $5 \mathrm{~min}$; $5 \mathrm{~min}$ later, the untrained site received two pretests at one per $5 \mathrm{~min}$. Then, there was a $1 \mathrm{hr}$ pretraining rest period during which the drug (APV or DNQX) was infused into the abdominal ganglion via the abdominal artery, after which habituation training of the trained site commenced. After the fourth (final) block of habituating stimuli was presented, there was a $2 \mathrm{hr}$ posttraining rest period to permit thorough washout of the APV-DNQX.

Data acquisition and evaluation. The force transducer used for measuring gill contractions (above) was connected through a bridge amplifier (ML110; ADInstruments) to a digital data acquisition system (MacLab 4s/2e; ADInstruments). The peak amplitude (maximum minimum) of each gill contraction was determined with the data acquisition system. The minimum was the baseline response recorded when the gill was in a relaxed state $1 \mathrm{sec}$ before stimulation. The maximum was the peak amplitude of the response within $20 \mathrm{sec}$ after the siphon stimulus. If the amplitude of the response declined by $\geq 80 \%$ from the initial peak within $20 \mathrm{sec}$ after the siphon stimulus, the response was considered

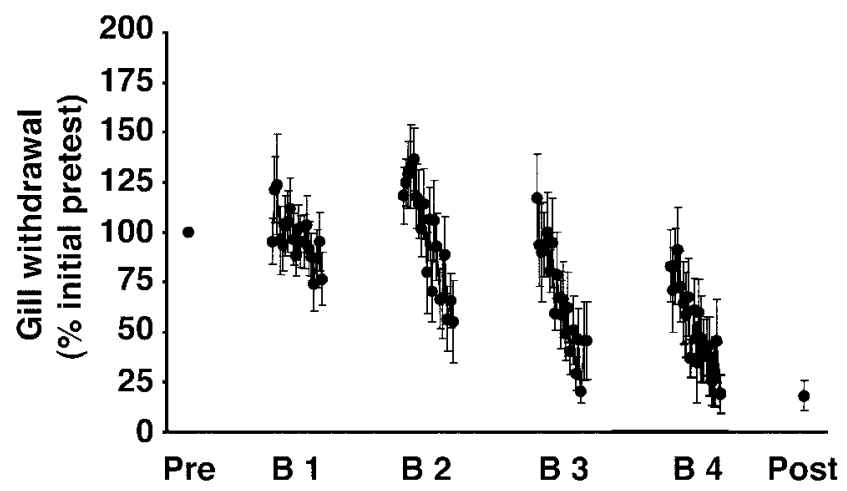

Figure 2. Long-lasting habituation of gill withdrawal in a reduced preparation. Gill withdrawal in reduced preparations that received spaced blocks of habituation training. Only one site on the siphon was stimulated in these experiments.

complete; subsequent responses within the $20 \mathrm{sec}$ period were not counted. Notice that, during training, the $30 \mathrm{sec}$ ISI was not always sufficient to permit the gill to fully relax after a response. In these cases, the minimum value for a particular response was the level of gill contraction before the onset of the training block.

Statistics. The pretest gill response in the experiments using the between-preparation design was the mean value for the three pretests. This value was set to be equal to $100 \%$, and the rest of the gill responses were normalized to the pretest value. There were also three posttests, and the mean of the three tests was used for the statistical comparisons. In the experiments using the within-preparation design, the pretest gill response for the trained site was also the mean value for the three pretests. The trained site also received three posttests. There were only a single pretest and a single posttest, however, for the untrained site in the withinpreparation experiments. The pretest value (mean of three pretests for the trained site or the single pretest value for the untrained site) was set to be equal to $100 \%$. The subsequent values for the gill responses to stimulation of the trained-untrained sites were normalized to $100 \%$. All responses are expressed as the normalized mean response \pm SEM. Nonparametric statistical tests were used for most comparisons. Wilcoxon tests were used for paired comparisons and Mann-Whitney tests were used for unpaired comparisons, unless otherwise indicated. All significance values reported represent two-tailed levels of significance.

\section{Results}

Long-lasting habituation of gill withdrawal in a reduced preparation

Our initial set of experiments used a between-preparation design (see Materials and Methods). There were two groups. One group (trained; $n=9$ ) received four blocks of habituating stimulation spaced at $90 \mathrm{~min}$ intervals. Each training block comprised 20 bouts of weak electrical stimulation of the siphon with a $30 \mathrm{sec}$ ISI. Another group (control; $n=13$ ) received only the pretest and posttest stimuli. When tested $6 \mathrm{hr}$ after the final (fourth) training block, gill withdrawal in the trained preparations exhibited significant habituation (mean posttest response, $34.6 \pm 5.9 \%$; $p<$ 0.004 for the comparison with the pretest value, which equaled $100 \%$ in this and all other experiments; see Materials and Methods) (Fig. 2). The magnitude of gill withdrawal did not change in the control preparations (mean posttest response, $91.5 \pm 12.3 \%$; $p>0.05$ for the comparison with the pretest response; data not shown). Furthermore, the difference between the posttest responses in the trained and control preparations was significant $(p<0.002)$. The lack of a significant difference in the magnitude of the gill reflex in control preparations during the experiments indicates that the decrement in the gill reflex in the trained group could not be attributable to deterioration in the health of the 
preparation over the $>12 \mathrm{hr}$ of the experiment. This suggests, in turn, that the training produced LLH in the trained group. It still might be argued, however, that the response decrement in the trained group was attributable to motor or muscle fatigue. We therefore modified our experimental protocol to exclude motormuscle fatigue as a possible explanation for the response decrement exhibited by the trained preparations.

\section{Reduced preparation exhibits site-specific long-lasting habituation of gill withdrawal}

In the rest of our experiments, we used a within-preparation design. Two stimulation sites on the siphon were used rather than one. One site (trained) received the spaced blocks of habituating stimuli, whereas the other site (untrained) received only the pretest and posttest stimuli. The testing and training protocol were similar to those in the between-preparation experiments (above), with the following exceptions. The untrained site received only a single pretest and a single posttest. Furthermore, the rest period between the end of the habituation training and the start of the posttest stimulation of the trained site was shortened from 6 to 1 hr. This was done to streamline the experiments. One hour after the end of training, the response of the gill to stimulation of the trained site was significantly reduced compared with the pretest response of the gill (mean posttest response, $64.8 \pm 4.2 \%$; $p<$ 0.003 for the comparison with the pretest response) (Fig. $3 A$ ). In contrast, the posttest response of gill to stimulation of the untrained site was significantly greater than the pretest response (mean posttest response, $115.1 \pm 7.3 \%$; $p<0.04$ for the comparison with the pretest response). This result suggests that the habituation training of the trained site induced modest sensitization of the untrained site within the same preparations. The response of the gill to stimulation of the trained site on the first of the three posttests $(77.4 \pm 7.6 \%)$ was also significantly less than the posttest response to stimulation of the untrained site $(p<$ $0.02 ; n=10$ ). (Notice that the interval between the pretests and posttests is identical for this comparison.) The difference between the posttest responses to the trained and untrained sites in these experiments cannot be ascribed to motor or muscle fatigue, because the same preparation was used to control for the effects of training on the neuromuscular components of the reflex. These results therefore demonstrate site-specific habituation of siphon-elicited gill withdrawal in a reduced preparation.

The amount of habituation was greater in the betweenpreparation experiments than in the within-preparation experiments ( $p<0.002$ for the comparison between the posttest value for the between-preparation experiments shown in Fig. 2 and the posttest value for trained-site stimulation in Fig. $3 A$ ). This difference may indicate that the longer interval between the termination of training and the posttests in the between-preparation experiments resulted in greater consolidation of LLH. However, because these two sets of experiments were performed at different times using animals from different shipments, it is also possible that the greater apparent habituation in the between-preparation experiments was attributable to a seasonal or animal shipment effect.

\section{Long-lasting habituation of gill withdrawal requires protein synthesis}

A hallmark of long-term memory across a range of preparations and different forms of learning is that it depends on the synthesis of new proteins (Davis and Squire, 1984; Goelet et al., 1986). To test whether LLH of gill withdrawal in our reduced preparation also exhibits a requirement for protein synthesis, we infused the
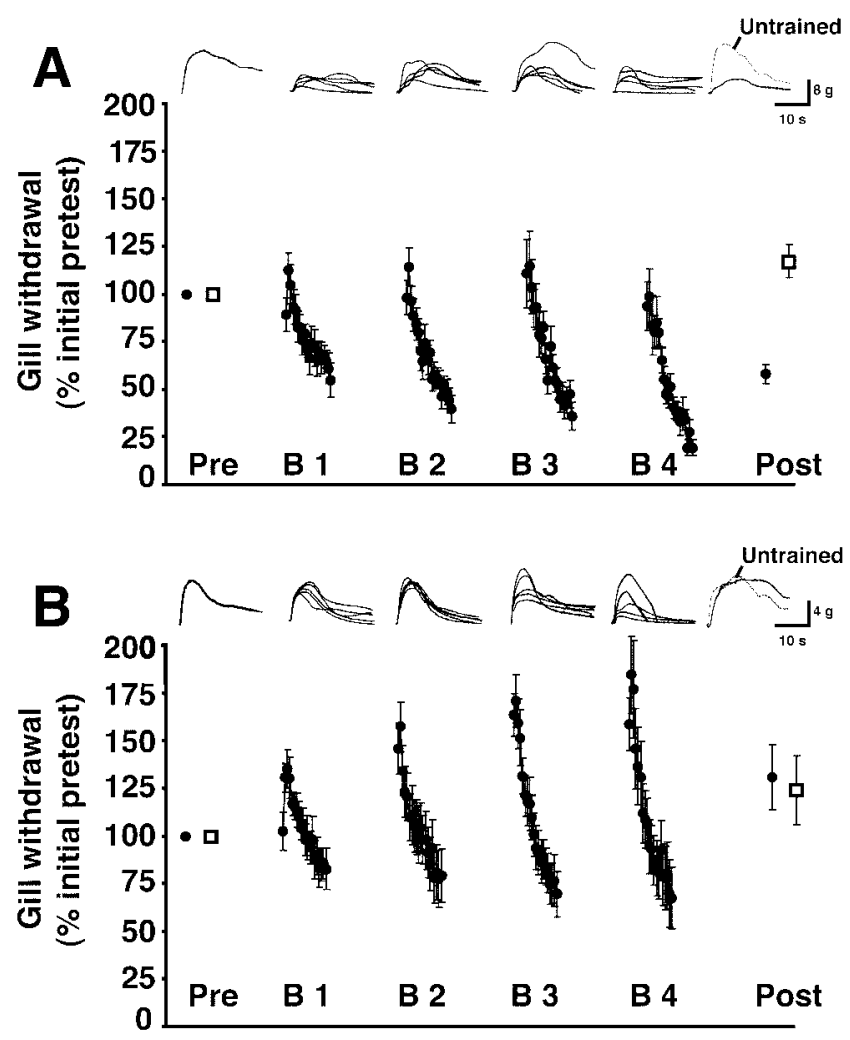

Figure 3. Effect of anisomycin on long-lasting habituation. $A$, Results from training in normal ASW. Averaged gill responses to trained-site stimulation in this and subsequent graphs are represented by filled circles; averaged responses to untrained-site stimulation are represented by open squares. Traces shown above the graph represent individual gill-withdrawal responses from a single experiment recorded using a force transducer (see Materials and Methods). From left to right, The response to the first trained-site pretest and the response to the untrained-site pretest are shown superimposed. (In this and subsequent figures, responses to trained-site stimulation are in black, and responses to untrained-site stimulation are in gray.) Next, five superimposed gill responses (those to stimuli 1, 3, 8, 13, and 20) from each of the four training blocks are presented sequentially. (These responses, except for the first and last responses of each block, were selected at random.) Finally, the response to the first trained-site posttest and the response to the untrained-site posttest are shown superimposed. $B$, Results from habituation training in the presence of the protein synthesis inhibitor anisomycin. Traces at top are the individual gill responses recorded during a single experiment. For details, see legend in $A$. Notice that the reflex exhibited significant interblock sensitization during training, as indicated by the increase in withdrawal responses to the first several stimuli of each training block.

protein synthesis inhibitor anisomycin (30 $\mu \mathrm{M}$ in ASW) into the abdominal ganglion before the start of an experiment. This concentration of anisomycin has been shown previously to inhibit protein synthesis by $>95 \%$ in the abdominal ganglion of Aplysia (Schwartz et al., 1971) and in sensory and motor neurons from the abdominal ganglion (Montarolo et al., 1986). The protein synthesis inhibitor was present throughout the experiment. Anisomycin blocked LLH of the withdrawal reflex to stimulation of the trained site. After habituation training in anisomycin, the response of the gill to stimulation of the trained siphon site was enhanced (posttest response, $126.7 \pm 14.6 \%$; $p>0.09$ for the comparison with the pretest response) (Fig. $3 B$ ), although this enhancement was not significant. The posttest response of the gill to stimulation of the untrained site $(121.0 \pm 15.3 \%)$ was also enhanced, although again not significantly $(p>0.1)$. Furthermore, the difference between the first posttest response to stimulation of the trained site $(160.5 \pm 18.7 \%)$ and the posttest response to stimulation of the untrained site was not significant $(p>0.09 ; n=9)$. A crucial comparison is that between the 
posttest values for trained site stimulation in the experiments of Figure 3, $A$ and $B$. This is because one might argue that the apparent blockade of LLH by anisomycin actually results from the fluctuations in the capacity for LLH that we observed among shipments of animals (see Materials and Methods). However, the two sets of experiments in Figure 3, $A$ and $B$, were performed at the same time using animals from the same shipments. Therefore, the experiment in Figure $3 A$ represents a control for the capability of the preparations used in the experiment in Figure $3 B$ to undergo habituation. The difference between the posttest response to trained-site stimulation in the experiments shown in Figure 3, $A$ and $B$, was highly significant $(p<0.001$ ). This result supports the notion that LLH was blocked in the experiments of Figure $3 B$ by the presence of the protein synthesis inhibitor.

\section{Long-lasting habituation of gill withdrawal requires protein phosphatase activity}

A potential cellular mechanism for LLH of gill withdrawal is long-term depression (LTD) of the monosynaptic connection between sensory and motor neurons in the abdominal ganglion (Lin and Glanzman, 1996). LTD of synapses in the mammalian hippocampus and cortex is attributable, in part, to activation of protein phosphatases (Mulkey et al., 1993; Bear and Abraham, 1996). To test for a possible role of protein phosphatase activity in habituation of gill withdrawal, we infused okadaic acid, a selective inhibitor of protein phosphatases 1 and 2A (PP1 and PP2A) (Cohen et al., 1990), into the abdominal ganglion. OA ( $1 \mu \mathrm{M}$ in ASW with $0.1 \%$ DMSO) was infused into the abdominal ganglion beginning $2 \mathrm{hr}$ before the first pretest and was present throughout the experiment. The presence of OA blocked LLH (Fig. 4A). The posttest response to stimulation of the trained site (108 $\pm 13.9 \%)$ was not significantly different from the pretest trained-site response $(p=1.0)$. Furthermore, there was no significant difference between the first posttest response to stimulation of the trained site $(118.4 \pm 12.1 \%)$ and the posttest response to stimulation of the untrained site in OA $(133.3 \pm 23.9 \%$; $p>0.8 ; n=$ 13). The OA did not appear to affect the response of the gill to stimulation of the untrained site over the course of the experiment. The posttest response to untrained-site stimulation was not significantly different from the pretest response $(p>0.5)$. To control for nonspecific actions of OA, we tested the effect of the control compound 1-nor-okadone ( $1 \mu \mathrm{M}$ in ASW with $0.1 \%$ DMSO), an analog of OA that does not inhibit phosphatases (Mulkey et al., 1993), on LLH of gill withdrawal (Fig. 4B). The experiments using 1-nor-okadone were performed at the same time as the experiments using $\mathrm{OA}$ and used animals from the same shipments. Infusion of 1-nor-okadone into the abdominal ganglion did not inhibit LLH. The posttest gill response to stimulation of the trained $(68.5 \pm 4.7 \%)$ site was significantly lower than the pretest response $(p<0.008 ; n=8)$. The 1 -nor-okadone treatment did not appear to affect the response of the gill to stimulation of the untrained site. The posttest response to stimulation of the untrained site $(117.6 \pm 22.1 \%)$ was not significantly different from the pretest response $(p>0.8)$. The posttest response of the gill to trained-site stimulation was significantly less in the 1-nor-okadone experiments than in the OA experiments $(p<0.03)$, a result that further supports the idea that protein phosphatase activity is essential for LLH.

\section{Long-lasting habituation of gill withdrawal requires activation of NMDA-type receptors}

The CNS of Aplysia possesses postsynaptic NMDA-type receptors (Dale and Kandel, 1993; Lin and Glanzman, 1994; Conrad et
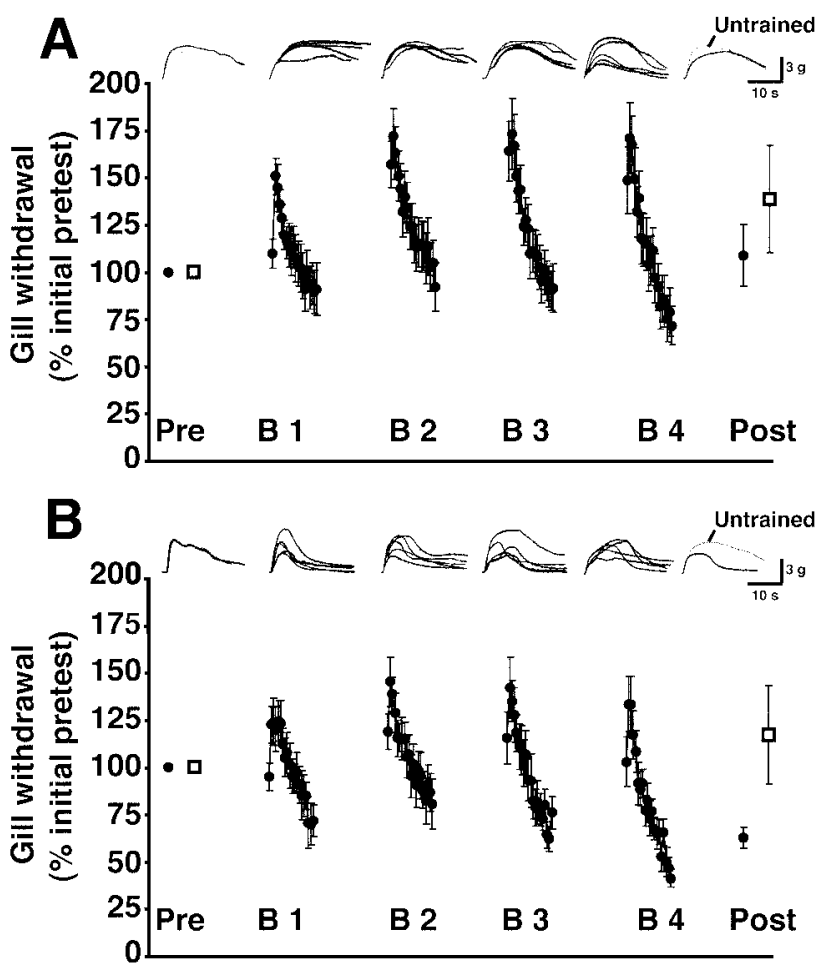

Figure 4. Effect of okadaic acid on long-lasting habituation. $A$, Results from training in the presence of okadaic acid. The drug was present throughout the experiments. Notice that the reflex exhibited interblock sensitization during training, as indicated by the increase in withdrawal responses to the first several stimuli of each training block. $B$, Results from habituation training in the presence of 1-nor-okadone. The drug was present throughout the experiments. Traces shown above the graphs in $A$ and $B$ are the individual gill responses recorded during a single experiment. For details, see the legend in Figure $3 A$.

al., 1999). Activation of postsynaptic NMDA-type receptors has been shown previously to be necessary for classical conditioning of the withdrawal reflex in Aplysia (Murphy and Glanzman, 1997, 1999; Antonov et al., 2003). We wanted to test whether activation of NMDA-type receptors was also required for LLH of the withdrawal reflex. For this test, we infused the specific NMDA receptor-antagonist APV (150 $\mu \mathrm{M}$ in ASW) (Collingridge et al., 1983; Lin and Glanzman, 1994; Conrad et al., 1999) into the abdominal ganglion. The drug was introduced into the abdominal artery $1 \mathrm{hr}$ before the first pretest and was present throughout the experiment. The presence of APV blocked LLH to the habituating stimuli (Fig. 5A). There was no significant difference between the pretest and posttest responses to stimulation of the trained site after training in APV (posttest response, $110.1 \pm$ $15.4 \%$ of the pretest response; $p>0.5 ; n=10$ ). Furthermore, there was no significant difference between the first posttest response to trained-site stimulation $(138 \pm 16 \%)$ and the posttest response to untrained-site stimulation $(123.6 \pm 15.1 \% ; p>0.5)$. APV treatment did not appear to affect gill withdrawal to stimulation of the untrained site, because it did not change significantly during the experiment $(p>0.2$ for the comparison between the pretest and posttest responses to untrained-site stimulation). We performed control experiments using animals from the same shipments that were used in the APV experiments. In the control experiments, the abdominal ganglion was continually infused with normal ASW. We observed normal LLH in the control experiments (Fig. $5 B$ ). The posttest response to stimulation of the trained site $(69.3 \pm 8 \%)$ was significantly decremented compared with the pretest response $(p<0.02 ; n=10)$. In contrast, there 

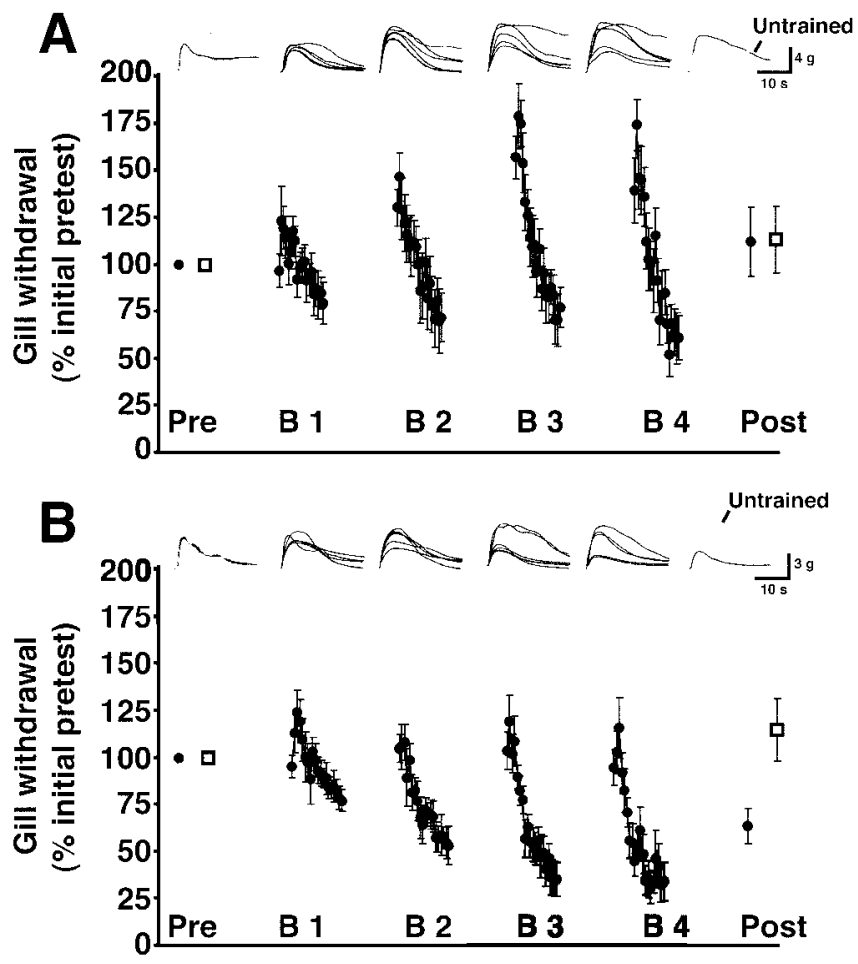

Figure 5. Effect of APV on long-lasting habituation. $A$, Results from training in the presence of APV. The drug was present throughout the experiments. (For results from experiments in which APV was present only during training, see Results.) Notice that the reflex exhibited significant interblock sensitization during training, as indicated by the increase in withdrawal responses to the first several stimuli of each training block. $B$, Results from habituation training in normal ASW without APV. Traces shown above the graphs in $A$ and $B$ are the individual gill responses recorded during a single experiment. For details, see the legend in Figure $3 A$.

was no change in the gill withdrawal to untrained-site stimulation (posttest response, $112.5 \pm 14.1 \% ; p>0.6$ for the comparison with the pretest response to untrained-site stimulation). Importantly, the difference between the posttest response to trained-site stimulation in the APV and control experiments was significant $(p<0.02)$.

In the above experiments, the APV was present during the pretests and posttests, as well as during training. To test whether our results would differ if the NMDA receptor antagonist were present only during training, we used a modified experimental protocol. This protocol was the same as that used for the DNQX experiments (below). We found that APV also blocked LLH when it was present in the abdominal ganglion only during training. The mean posttest response to stimulation of the trained site after training in APV did not differ significantly from the pretest response (posttest response, $120 \pm 20.7 \%$; $p>0.4$ for the comparison with the pretest response; one-sample $t$ test; $n=4$; data not shown), nor was the mean posttest response to stimulation of the untrained site in the APV experiments significantly different from the pretest response (posttest response, $106 \pm 11.4 \%$; $p>$ 0.5 for the comparison with the pretest response). There was also no significant difference between the mean posttest responses to trained-site and untrained-site stimulation after training in APV ( $p>0.5$; paired $t$ test). In contrast, in control experiments performed on animals from the same shipments that were used for the APV experiments, we observed LLH of the trained-site response. The mean posttest response to stimulation of the trained site after training in ASW was significantly habituated (posttest response, $47.5 \pm 6.9 \%$; $p<0.005$ for the comparison with the pretest response; one-sample $t$ test; $n=4$; data not shown). Furthermore, the mean posttest response to trained-site stimulation was significantly less than that to untrained-site stimulation after training in ASW ( $p<0.05$; paired $t$ test). The mean posttest response to stimulation of the untrained site in the ASW experiments was not significantly different from the pretest response (posttest response, $105.8 \pm 16.7 \%$; $p>0.7$ for the comparison with the pretest response to untrained-site stimulation). Significantly, the posttest response to trained-site stimulation was more habituated after training in ASW than after training in APV $(p<$ 0.03 ). These results support the conclusion from our other experiments involving APV (Fig. 5) that the presence of an NMDA receptor antagonist during training disrupts LLH.

\section{Long-lasting habituation of gill withdrawal requires activation of AMPA-type receptors}

It has been long believed that homosynaptic depression of transmission between the sensory and motor neurons in the abdominal ganglion, an exclusively presynaptic form of plasticity (Castellucci and Kandel, 1974; Armitage and Siegelbaum, 1998), is a cellular mechanism of habituation of the withdrawal reflex (Kandel et al., 1975). Armitage and Siegelbaum (1998) have shown that application of DNQX, an antagonist of AMPA-type receptors in Aplysia motor neurons (Dale and Kandel, 1993; Chitwood et al., 2001), does not disrupt short-term homosynaptic depression, although DNQX blocks transmission at the sensorimotor synapse. They also found that repeated application of glutamate, the sensory neuron transmitter (Dale and Kandel, 1993; Levenson et al., 2000), to the motor neuron did not depress the synaptically evoked sensorimotor EPSP. On the basis of their results Armitage and Siegelbaum concluded that activation of postsynaptic glutamate receptors was neither necessary nor sufficient for the induction of homosynaptic depression of sensorimotor synapses.

We tested whether activation of postsynaptic AMPA-type receptors was necessary for the induction of LLH. We infused DNQX (500 $\mu \mathrm{M}$ in ASW with $0.2 \%$ DMSO) into the abdominal ganglion during habituation training. To give adequate time for the drug to be washed in and washed out of the abdominal ganglion, we modified our basic experimental protocol. In these experiments, there was a $1 \mathrm{hr}$ rest period between the end of the pretests and the onset of habituation training. DNQX was introduced into the abdominal artery immediately after the pretests and was present throughout the training. After the last block of training, there was a $2 \mathrm{hr}$ rest period during which the DNQX was washed out of the abdominal ganglion (using ASW with $0.2 \%$ DMSO). Another modification in the protocol was that there were two pretests to both the trained and untrained sites (the pretests were administered alternately to the two sites at $5 \mathrm{~min}$ intervals). Each of the sites also received two posttests (also administered alternately to the sites at 5 min intervals). The presence of DNQX during habituation training blocked the induction of LLH (Fig. 6A). The mean posttest response of the gill to trained-site stimulation $(108.4 \pm 10.6 \%)$ was not significantly different from the pretest response $(p>0.6 ; n=6)$. Furthermore, the mean posttest response to trained-site stimulation was not significantly different from that to untrained-site stimulation (140.6 $\pm 23.5 \% ; p>0.2$ ). Control experiments were performed using a protocol identical to that used in the DNQX experiments, as well as animals from the same shipments. The abdominal artery was infused with ASW and 0.2\% DMSO throughout the control experiments. The habituation training induced LLH of gill withdrawal in the control experiments (Fig. 6B). The mean 

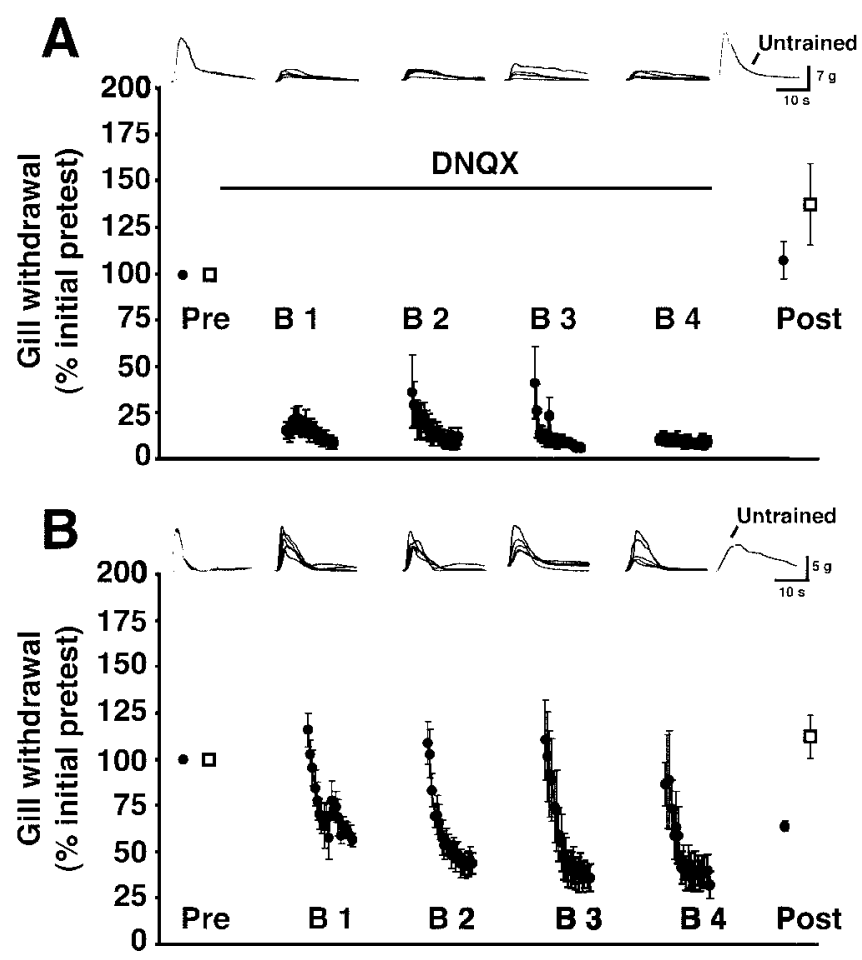

Figure 6. Effect of DNQX on long-lasting habituation. $A$, Results from training in the presence of DNQX. The drug was present only during the training, as indicated by the black line. Notice that the responses evoked during training in the presence of DNQX were greatly reduced but not completely eliminated (for discussion, see Results). $B$, Results from habituation training in ASW with $0.2 \%$ DMSO. Traces shown above the graphs in $A$ and $B$ are the individual gill responses recorded during a single experiment. The set of traces shown with each graph are from one preparation. For details, see the legend in Figure $3 A$.

posttest response to stimulation of the trained site was $61.9 \pm$ $3.2 \%(p<0.04 ; n=6)$. Also, the mean posttest response to trained-site stimulation was significantly less than the mean posttest response to untrained-site stimulation (113.2 $\pm 12.2 \%$; $p<$ 0.04 ) in the control experiments. Finally, the mean posttest response to trained-site stimulation was significantly greater in the DNQX experiments than in the control experiments $(p<0.003)$.

Notice that, although the DNQX significantly reduced gill withdrawal to the training stimuli, the blockade of the response was incomplete (Fig. 6A). The concentration of DNQX that we used was approximately an order of magnitude greater than that shown in previous studies to produce a $>90 \%$ blockade of synaptic transmission at sensorimotor synapses (Dale and Kandel, 1993; Armitage and Siegelbaum, 1998). The reduced gill responses observed during training in the presence of DNQX might reflect the contribution of peripheral sensorimotor synapses to the reflex (Peretz and Estes, 1974; Bailey et al., 1979; Frost et al., 1997). Such synapses would not have been affected by the DNQX, because the infusion of this drug was limited to the abdominal artery, which supplies the abdominal ganglion. Early work indicated that the peripheral nervous system alone can support habituation of gill withdrawal in Aplysia (Peretz, 1970; Lukowiak and Jacklet, 1972; Peretz and Moller, 1974; Lukowiak and Jacklet, 1975; Peretz et al., 1976). If the remnant of the withdrawal response apparent during training in DNQX is indeed mediated by the peripheral nervous system, our data confirm that the isolated peripheral nervous system can mediate short-term habituation (STH) of gill withdrawal, at least to some extent. An alternative explanation for the inability of the DNQX infusion to completely eliminate gill withdrawal in our experiments is that the drug did not have access to all of the sensorimotor synapses in the abdominal ganglion.

In all of the above within-preparation experiments, we excluded from our data analysis those preparations in which the posttest response to stimulation of the untrained siphon site was at least $80 \%$ of the pretest response (see Materials and Methods). We did this because we wanted to exclude results from preparations that had deteriorated. However, because it could be argued that we thereby biased our results, we reanalyzed all of our within-preparation data after including the data from the excluded preparations $(n=6)$. Adding the excluded preparations to our data did not alter any of our main results.

\section{Discussion}

The role of protein synthesis in LLH in Aplysia

The present data demonstrate that prolonged habituation of the withdrawal reflex in Aplysia differs from STH in its requirement for protein synthesis. The lack of a requirement by STH for protein synthesis agrees with results from a previous study (Schwartz et al., 1971). Our study, however, represents the first demonstration that prolonged habituation of the withdrawal reflex requires protein synthesis. A requirement for protein synthesis has been also been observed for LTH of reflexive responses in the nematode worm Caenorhabditis elegans (Rose and Rankin, 2001; Rose et al., 2002) and the crab Chasmagnathus (Pedreira et al., 1996; Hermitte et al., 1999), as well as for LTH of the responsiveness to distress cries in mice (Squire and Becker, 1975).

We do not yet know whether LLH depends on RNA synthesis, as well as protein synthesis. An intermediate form of sensitization has been described recently in Aplysia (Sutton et al., 2001). Intermediate sensitization differs mechanistically from short-term sensitization in its dependence on protein synthesis. Intermediate sensitization differs from long-term sensitization (Frost et al., 1985; Castellucci et al., 1989) in that long-term sensitization requires RNA synthesis, whereas the intermediate form does not. Possibly, LLH represents an intermediate phase of habituation, analogous to intermediate sensitization.

\section{Implications of site specificity of LLH for localizing the cellular site of plasticity}

Because LLH of siphon-elicited gill withdrawal exhibits site specificity, the behavioral decrement cannot be ascribed to motor or muscle fatigue or to a gradual deterioration in the health of the preparations. The demonstration of site specificity also places certain limits on the locus of the cellular changes that underlie LLH. For example, LLH cannot be attributable to a general, staterelated change within the CNS. Furthermore, if LLH is attributable, at least partly, to enhanced inhibitory input to the withdrawal circuitry, then the inhibitory input must be somewhat specific with respect to sensorimotor pathways within the abdominal ganglion.

Stopfer et al. (1996) have shown previously that STH and LTH (lasting $24 \mathrm{hr}$ ) of the tail-elicited siphon-withdrawal reflex exhibit site specificity. Stopfer et al. argued that site-specific habituation in their experiments proved that "the cellular sites of plasticity must exist upstream from the motor neurons," because the siphon motor neurons receive common interneuronal input from both sides of the tail. However, the motor neurons might well be a site of plasticity in our experiments. Site specificity of habituation can be maintained with the cellular locus of plasticity in the motor neurons if the learning-related changes are restricted to specific postsynaptic sites. For example, both NMDA 
receptor-dependent long-term potentiation and LTD in the mammalian CNS exhibit input specificity, although these forms of plasticity are induced and, at least in part, expressed postsynaptically (Bliss and Collingridge, 1993; Bear and Linden, 2001).

\section{Stimuli that induce habituation of the gill-withdrawal reflex appear to activate sensitizing cellular processes as well} In 1970, Groves and Thompson proposed the "dual process" theory of habituation. According to this theory, stimuli that induce habituation in a particular stimulus-response pathway also recruit an independent, incremental (sensitizing) process that increases the state of excitation in an organism. The final response strength in the Groves and Thompson model is determined by the net outcome of the countervailing processes of habituation and sensitization. Our data provide support for the dual-process theory of habituation. Treatments that blocked LLH (inhibition of protein synthesis, inhibition of protein phosphatase activity, and antagonism of NMDA-type receptors) also revealed the effects of a sensitizing process elicited by habituation training. This sensitizing process is apparent in the tendency of the withdrawal responses to trained-site stimulation to increase in strength over the course of habituation training (Figs. $3 B, 4 A, 5 A$ ).

\section{Homosynaptic, exclusively presynaptic, depression of the sensorimotor synapses is not the mechanism of LLH in Aplysia}

The data from the present study indicate that homosynaptic depression of sensorimotor synapses, an exclusively presynaptic form of plasticity (Castellucci and Kandel, 1974; Armitage and Siegelbaum, 1998; Royer et al., 2000; Gover et al., 2002), is insufficient for LLH. In most of the experiments that used pharmacological blocking agents, there was a dissociation between the effect of the agents on LLH and their effect on STH. Specifically, neither anisomycin (Fig. 3B), OA (Fig. 4A), nor APV (Fig. 5A) blocked STH, yet each blocked LTH. Given that STH is mediated primarily by homosynaptic depression (Frost et al., 1997; Antonov et al., 1999), our results imply that homosynaptic depression of sensorimotor synapses is insufficient for the induction of prolonged habituation. The DNQX experiment provides compelling evidence that homosynaptic depression is not the mechanism of LLH. This is because application of DNQX to the sensorimotor synapse does not alter short-term homosynaptic depression (Armitage and Siegelbaum, 1998), whereas application of DNQX to the abdominal ganglion blocked LLH (Fig. 6A).

An alternative explanation for our DNQX result is that the long-term cellular change that underlies LLH occurs exclusively, or mostly, at synapses between interneurons and motor neurons (below). According to this hypothesis, the DNQX blocked synaptic transmission at the sensory neuron-to-interneuron synapses. This, in turn, prevented activation of the interneurons, thereby disrupting long-term plastic changes at the interneuronto-motor neuron synapses. Although this explanation cannot be ruled out by our data, it seems unlikely given that LTH of gill withdrawal is accompanied by significant long-term depression of sensorimotor synapses in the abdominal ganglion (Castellucci et al., 1978).

The role of postsynaptic glutamate receptor activation in LLH Our data indicate that activation of postsynaptic glutamate receptors (Dale and Kandel, 1993; Trudeau and Castellucci, 1993; Lin and Glanzman, 1994; Conrad et al., 1999; Yung et al., 2002) is critical for the induction of LLH. Antonov et al. (2003) have reported recently that high concentrations of DNQX can signif- icantly reduce the NMDA-type receptor component, as well as the AMPA-type receptor component, of the sensorimotor EPSP (Antonov et al., 2003). Possibly, the critical action of DNQX in our experiments was on the NMDA type rather than the AMPA type. Future experiments with more selective AMPA receptor antagonists can resolve this issue. Interestingly, a study of LTH of the tap withdrawal response in C. elegans has found that mutant worms lacking a homolog of the vertebrate AMPA receptor [ $g l r-1$ (glutamate receptor 1) mutants] exhibit normal STH to spaced blocks of taps but do not show LTH (Rose et al., 2001).

\section{Does an LTD-related synaptic mechanism mediate LLH in Aplysia?}

The cellular processes essential for LLH induction are reminiscent of those necessary for the induction of homosynaptic LTD of synapses in the mammalian hippocampus and cortex (Mulkey and Malenka, 1992; Mulkey et al., 1993; Kirkwood and Bear, 1994). According to a current model of LTD (Bear and Linden, 2001), low-frequency stimulation (LFS) of afferent inputs causes modest activation of postsynaptic NMDA receptors, resulting in a moderate influx of $\mathrm{Ca}^{2+}$ into the dendrites of the postsynaptic cell. This postsynaptic influx of $\mathrm{Ca}^{2+}$, in turn, activates calcineurin (PP2B), which releases PP1 from inhibition, thereby permitting PP1 to become active. Activation of these protein phosphatases leads to long-term synaptic weakening. The precise pathways by which activation of $\mathrm{PP} 2 \mathrm{~B}$ and $\mathrm{PP} 1$ produces LTD are not completely understood, but it is believed that the protein phosphatase cascade causes the endocytosis of AMPA receptors, thereby resulting in fewer receptors in the postsynaptic membrane (Carroll et al., 2001).

An LTD-like form of synaptic plasticity has been reported previously for Aplysia sensorimotor synapses (Lin and Glanzman, 1996). Like homosynaptic LTD of hippocampal synapses (Mulkey and Malenka, 1992), LTD of sensorimotor synapses depends on a postsynaptic rise in $\mathrm{Ca}^{2+}$ because it is blocked by postsynaptic injection of the rapid $\mathrm{Ca}^{2+}$ chelator BAPTA before the onset of LFS. Whether this form of LTD of Aplysia synapses also resembles LTD of mammalian synapses in its requirement for activation of protein phosphatases and NMDA receptors is not yet known.

\section{The potential role of interneurons in LLH of siphon-elicited gill withdrawal}

The present data do not rule out contributions from changes in interneuronal pathways. Indeed, the previous failure to obtain long-term (24 hr) depression of in vitro sensorimotor synapses using various patterns of homosynaptic stimulation, together with the demonstration that repeated applications of the inhibitory peptide FMRFamide (Piomelli et al., 1987) can induce longterm depression (Montarolo et al., 1988), suggest that contributions from heterosynaptic mechanisms might play a role in LLH. A potential link between our finding that inhibition of protein phosphatase activity can block the induction of LLH (Fig. 4A) and a possible role for FMRFamide in LLH is provided by evidence that at least some neuronal actions of FMRFamide within the CNS of Aplysia are mediated by activation of protein phosphatases (Sweatt et al., 1989; Ichinose and Byrne, 1991).

\section{Conclusion}

Habituation of the withdrawal reflex in Aplysia is frequently regarded as one of the simplest forms of learning with respect to its underlying cellular mechanisms. However, the present data demonstrate that our current understanding of habituation of the withdrawal reflex is very incomplete. Postsynaptic mechanisms 
play a far more important role in habituation than has been heretofore appreciated. In particular, our data raise the possibility that an LTD-like synaptic mechanism may mediate this simple form of invertebrate learning.

\section{References}

Antonov I, Kandel ER, Hawkins RD (1999) The contribution of facilitation of monosynaptic PSPs to dishabituation and sensitization of the Aplysia siphon withdrawal reflex. J Neurosci 19:10438-10450.

Antonov I, Antonova I, Kandel ER, Hawkins RD (2003) Activity-dependent presynaptic facilitation and Hebbian LTP are both required and interact during classical conditioning in Aplysia. Neuron 37:135-147.

Armitage BA, Siegelbaum SA (1998) Presynaptic induction and expression of homosynaptic depression at Aplysia sensorimotor neuron synapses. J Neurosci 18:8770-8779.

Bailey CH, Chen M (1983) Morphological basis of long-term habituation and sensitization in Aplysia. Science 220:91-93.

Bailey CH, Chen M (1988) Long-term memory in Aplysia modulates the total number of varicosities of single identified sensory neurons. Proc Natl Acad Sci USA 85:2373-2377.

Bailey CH, Castellucci VF, Koester J, Kandel ER (1979) Cellular studies of peripheral neurons in siphon skin of Aplysia californica. J Neurophysiol 42:530-557.

Bear MF, Abraham WC (1996) Long-term depression in hippocampus. Annu Rev Neurosci 19:437-462.

Bear MF, Linden DJ (2001) The mechanisms and meaning of long-term synaptic depression in the mammalian brain. In: Synapses (Cowan WM, Südhof TC, Stevens CF, eds), p 455-517. Baltimore: Johns Hopkins UP.

Bliss TVP, Collingridge GL (1993) A synaptic model of memory: long-term potentiation in the hippocampus. Nature 361:31-39.

Carew TJ, Kandel ER (1973) Acquisition and retention of long-term habituation in Aplysia: correlation of behavioral and cellular processes. Science 182:1158-1160

Carew TJ, Pinsker HM, Kandel ER (1972) Long-term habituation of a defensive withdrawal reflex in Aplysia. Science 175:451-454.

Carroll RC, Beattie EC, von Zastrow M, Malenka RC (2001) Role of AMPA receptor endocytosis in synaptic plasticity. Nat Rev Neurosci 2:315-324.

Castellucci VF, Kandel ER (1974) A quantal analysis of the synaptic depression underlying habituation of the gill-withdrawal reflex in Aplysia. Proc Natl Acad Sci USA 71:5004-5008.

Castellucci VF, Carew TJ, Kandel ER (1978) Cellular analysis of long-term habituation of the gill-withdrawal reflex of Aplysia californica. Science 202:1306-1308.

Castellucci VF, Blumenfeld H, Goelet P, Kandel ER (1989) Inhibitor of protein synthesis blocks long-term behavioral sensitization in the isolated gill-withdrawal reflex of Aplysia. J Neurobiol 20:1-9.

Chitwood RA, Li Q, Glanzman DL (2001) Serotonin facilitates AMPA-type responses in isolated siphon motor neurons of Aplysia in culture. J Physiol (Lond) 534:501-510.

Cohen P, Holmes CF, Tsukitani Y (1990) Okadaic acid: a new probe for the study of cellular regulation. Trends Biochem Sci 15:98-102.

Collingridge GL, Kehl SJ, McLennan H (1983) Excitatory amino acids in synaptic transmission in the Schaffer collateral-commissural pathway of the rat hippocampus. J Physiol (Lond) 334:33-46.

Conrad P, Wu F, Schacher S (1999) Changes in functional glutamate receptors on a postsynaptic neuron accompany formation and maturation of an identified synapse. J Neurobiol 39:237-248.

Dale N, Kandel ER (1993) L-glutamate may be the fast excitatory transmitter of Aplysia sensory neurons. Proc Natl Acad Sci USA 90:7163-7167.

Davis HP, Squire LR (1984) Protein synthesis and memory: a review. Psychol Bull 96:518-559.

Ezzeddine Y, Glanzman DL (2001) Role of protein phosphatases and $\mathrm{N}$-methyl-D-aspartate-type receptors in long-term habituation of the gillwithdrawal reflex in Aplysia. Soc Neurosci Abstr 27:954.11.

Ezzeddine Y, Glanzman DL (2002) Role of protein synthesis and protein kinase activity in long-term habiutation of the gill-withdrawal reflex in Aplysia. Soc Neurosci Abstr 28:376.6.

Frost L, Kaplan SW, Cohen TE, Henzi V, Kandel ER, Hawkins RD (1997) A simplified preparation for relating cellular events to behavior: contribution of LE and unidentified siphon sensory neurons to mediation and habituation of the Aplysia gill- and siphon-withdrawal reflex. J Neurosci 17:2900-2913.
Frost WN, Castellucci VF, Hawkins RD, Kandel ER (1985) Monosynaptic connections made by the sensory neurons of the gill- and siphonwithdrawal reflex in Aplysia participate in the storage of long-term memory for sensitization. Proc Natl Acad Sci USA 82:8266-8269.

Geer JH (1966) Effect of interstimulus intervals and rest-periol length upon habituation of the orienting response. J Exp Psychol 72:617-619.

Goelet P, Castellucci VF, Schacher S, Kandel ER (1986) The long and the short of long-term memory-a molecular framework. Nature 322:419-422.

Gover TD, Jiang XY, Abrams TW (2002) Persistent, exocytosisindependent silencing of release sites underlies homosynaptic depression at sensory synapses in Aplysia. J Neurosci 22:1942-1955.

Groves PM, Thompson RF (1970) Habituation: a dual-process theory. Psychol Rev 77:419-450.

Hermitte G, Pedreira ME, Tomsic D, Maldonado H (1999) Context shift and protein synthesis inhibition disrupt long-term habituation after spaced, but not massed, training in the crab Chasmagnathus. Neurobiol Learn Mem 71:34-49.

Ichinose M, Byrne JH (1991) Role of protein phosphatases in the modulation of neuronal membrane currents. Brain Res 549:146-150.

Kandel ER (1978) A cell-biological approach to learning and memory. Bethesda, MD: Society for Neuroscience.

Kandel ER, Brunelli M, Byrne J, Castellucci V (1975) A common presynaptic locus for the synaptic changes underlying short-term habituation and sensitization of the gill-withdrawal reflex in Aplysia. Cold Spring Harbor Symp Quant Biol 40:465-482.

Kirkwood A, Bear MF (1994) Homosynaptic long-term depression in the visual cortex. J Neurosci 14:3404-3412.

Krasne FB (1976) Invertebrate systems as a means of gaining insight into the nature of learning and memory. In: Neural mechanisms of learning and memory (Rosenzweig MR, Bennett EL, eds), pp 401-429. Cambridge, MA: MIT.

Krasne FB, Glanzman DL (1995) What we can learn from invertebrate learning. Annu Rev Psychol 46:585-624.

Krasne FB, Teshiba TM (1995) Habituation of an invertebrate escape reflex due to modulation by higher centers rather than local events. Proc Natl Acad Sci USA 92:3362-3366.

Levenson J, Sherry DM, Dryer L, Chin J, Byrne JH, Eskin A (2000) Localization of glutamate and glutamate transporters in the sensory neurons of Aplysia. J Comp Neurol 423:121-131.

Lin XY, Glanzman DL (1994) Hebbian induction of long-term potentiation of Aplysia sensorimotor synapses: partial requirement for activation of an NMDA-related receptor. Proc R Soc Lond B Biol Sci 255:215-221.

Lin XY, Glanzman DL (1996) Long-term depression of Aplysia sensorimotor synapses in cell culture: inductive role of a rise in postsynaptic calcium. J Neurophysiol 76:2111-2114.

Lukowiak K, Jacklet JW (1972) Habituation and dishabituation: interactions between peripheral and central nervous systems in Aplysia. Science 178:1306-1308.

Lukowiak K, Jacklet J (1975) Habituation and dishabituation mediated by the peripheral and central neural circuits of the siphon of Aplysia. J Neurobiol 6:183-200.

Montarolo PG, Goelet P, Castellucci VF, Morgan J, Kandel ER, Schacher S (1986) A critical period for macromolecular synthesis in long-term heterosynaptic facilitation in Aplysia. Science 234:1249-1254.

Montarolo PG, Kandel ER, Schacher S (1988) Long-term heterosynaptic inhibition in Aplysia. Nature 333:171-174.

Mulkey RM, Malenka RC (1992) Mechanisms underlying induction of homosynaptic long-term depression in area CAl of the hippocampus. Neuron 9:967-975.

Mulkey RM, Herron CE, Malenka RC (1993) An essential role for protein phosphatases in hippocampal long-term depression. Science 261:1051-1055.

Murphy GG, Glanzman DL (1997) Mediation of classical conditioning in Aplysia californica by LTP of sensorimotor synapses. Science 278:467-471.

Murphy GG, Glanzman DL (1999) Cellular analog of differential classical conditioning in Aplysia: disruption by the NMDA receptor-antagonist DL-2-amino-5-phosphonovalerate. J Neurosci 19:10595-10602.

Pedreira ME, Dimant B, Maldonado H (1996) Inhibitors of protein and RNA synthesis block context memory and long-term habituation in the crab Chasmagnathus. Pharmacol Biochem Behav 54:611-617.

Peretz B (1970) Habituation and dishabituation in the absence of a central nervous system. Science 169:379-381. 
Peretz B, Estes J (1974) Histology and histochemistry of the peripheral neural plexus in the Aplysia gill. J Neurobiol 5:3-19.

Peretz B, Moller R (1974) Control of habituation of the withdrawal reflex by the gill ganglion in Aplysia. J Neurobiol 5:191-212.

Peretz B, Jacklet JW, Lukowi K (1976) Habituation of reflexes in Aplysia: contribution of the peripheral and central nervous systems. Science 191:396-399.

Piomelli D, Volterra A, Dale N, Siegelbaum SA, Kandel ER, Schwartz JH, Belardetti F (1987) Lipoxygenase metabolites of arachidonic acid as second messengers for presynaptic inhibition of Aplysia sensory cells. Nature 328:38-43.

Rankin CH, Beck CD, Chiba CM (1990) Caenorhabditis elegans: a new model system for the study of learning and memory. Behav Brain Res 37:89-92.

Rose JK, Rankin CH (2001) Analyses of habituation in Caenorhabditis elegans. Learn Mem 8:63-69.

Rose JK, Chen S, Kaun KR, Rankin CH (2001) Glutamate and AMPAreceptor function are necessary for long-term memory in C. elegans. Soc Neurosci Abstr 27:87.9.

Rose JK, Kaun KR, Rankin CH (2002) A new group-training procedure for habituation demonstrates that presynaptic glutamate release contributes to long-term memory in Caenorhabditis elegans. Learn Mem 9:130-137.

Royer S, Coulson RL, Klein M (2000) Switching off and on of synaptic sites at Aplysia sensorimotor synapses. J Neurosci 20:626-638.

Schwartz JH, Castellucci VF, Kandel ER (1971) Functioning of identified neurons and synapses in abdominal ganglion of Aplysia in absence of protein synthesis. J Neurophysiol 34:939-953.
Squire LR, Becker CK (1975) Inhibition of cerebral protein synthesis impairs long-term habituation. Brain Res 97:367-372.

Stopfer M, Carew TJ (1996) Heterosynaptic facilitation of tail sensory neuron synaptic transmission during habituation in tail-induced tail and siphon withdrawal reflexes of Aplysia. J Neurosci 16:4933-4948.

Stopfer M, Chen X, Tai YT, Huang GS, Carew TJ (1996) Site specificity of short-term and long-term habituation in the tail-elicited siphon withdrawal reflex of Aplysia. J Neurosci 16:4923-4932.

Sutton MA, Masters SE, Bagnall MW, Carew TJ (2001) Molecular mechanisms underlying a unique intermediate phase of memory in Aplysia. Neuron 31:143-154.

Sweatt JD, Volterra A, Edmonds B, Karl KA, Siegelbaum SA, Kandel ER (1989) FMRFamide reverses protein phosphorylation produced by 5-HT and cAMP in Aplysia sensory neurons. Nature 342:275-278.

Thompson RF, Spencer WA (1966) Habituation: a model phenomenon for the study of neuronal substrates of behavior. Psychol Rev 73:16-43.

Trudeau LE, Castellucci VF (1993) Excitatory amino acid neurotransmission at sensory-motor and interneuronal synapses of Aplysia californica. J Neurophysiol 70:1221-1230.

Yung I, Chun S, Kapya E, Moroz LL, Martin KC, Boulter J, Glanzman DL (2002) Cloning of glutamate receptors from the central nervous system of Aplysia. Soc Neurosci Abstr 28:376.7.

Zucker RS (1972) Crayfish escape behavior and central synapses. II. Physiological mechanisms underlying behavioral habituation. J Neurophysiol 35:621-637. 